\title{
Euler potentials' discontinuity in the presence of field line-aligned currents
}

\author{
HUANG TianSen ${ }^{1,2^{*}} \&$ WU DeJin ${ }^{1}$ \\ ${ }^{1}$ Purple Mountain Observatory, Chinese Academy of Sciences, Nanjing 210008, China; \\ ${ }^{2}$ Solar Observatory, College of Arts and Sciences, Prairie View A\&M University, Houston, TX 77446, USA
}

Received November 17, 2011; accepted February 3, 2012; published online March 8, 2012

\begin{abstract}
Since their use in the study of charged particle motion in the 1960s, Euler potentials $(\alpha, \beta)$ have been widely employed as magnetic field coordinates in both space plasma and fusion plasma studies. People related them to magnetic vector potential $\boldsymbol{A}$ via the relation $\boldsymbol{A}=\alpha \nabla \beta$, subject to gauge condition $\boldsymbol{A} \cdot \boldsymbol{B}=0$ ( $\boldsymbol{B}$ is the magnetic induction). For a given magnetic field, the Euler potentials are often constructed with the relation $\boldsymbol{B} \cdot \Delta S=\Delta \alpha \Delta \beta$ on a surface that crosses the field lines, where $\Delta S$ is the area-element surrounding by two line-elements corresponding to the changes in $\alpha$ and $\beta$, then mapping the values of $\alpha$ and $\beta$ along field lines into space. In this short paper, we show that in the presence of field line-aligned currents, the mapping does not work and the orthogonality gauge condition is not satisfied.
\end{abstract}

plasmas, space physics, Euler potential, drift motion

Citation: Huang T S, Wu D J. Euler potentials' discontinuity in the presence of field line-aligned currents. Chin Sci Bull, 2012, 57: 1436-1437, doi: 10.1007/ s11434-012-5043-0

By representing magnetic field as $\boldsymbol{B}=\nabla \alpha \times \nabla \beta$, Grad [1] was the first to use two scalar functions $\alpha$ and $\beta$ as the field line coordinates, and referred to them as Euler potentials [2]. In Northrop and Teller's famous paper [3], the $(\alpha, \beta)$ coordinates were used in proving the second adiabatic invariant and deriving the dynamic equations of a charged particle:

$$
\langle\dot{\alpha}\rangle=-\frac{1}{q} \frac{\partial K}{\partial \beta},\langle\dot{\beta}\rangle=\frac{1}{q} \frac{\partial K}{\partial \alpha},
$$

where $q$ is the particle's charge and $K$ is sum of the particle's kinetic energy and electric potential energy plus a quantity $q \alpha \partial \beta / \partial t$. Euler potentials have since been widely employed in studying space plasma physics (e.g. [4-6]). The potentials are also used in the study of fusion plasma physics under the name of Clebsch coordinates [7]. The applicability of Euler potentials as magnetic field coordinates has been presented [4,8], but no strict proof has been presented. Below, we give an example to show that in the

\footnotetext{
*Corresponding author (email: tshuang@pvamu.edu)
}

presence of field line-aligned current, the Euler potentials are discontinuous whereas the magnetic field $\boldsymbol{B}$ and the vector potential $\boldsymbol{A}$ have no discontinuity.

\section{Some concerns about the application of Euler potentials}

In his mathematics text book [9], Phillips showed that a solenoidal and continuously differentiable vector function, such as a magnetic field $\boldsymbol{B}$ can be expressed as a crossproduct of two gradients, $\boldsymbol{B}=\nabla \alpha \times \nabla \beta$. Northrop [8] gave one simple argument for the introduction of $\alpha$ and $\beta$ as magnetic field line coordinates; that is roughly the same as Phillips' proof. Both Phillips' proof and Northrop's argument invoked the assumption that all magnetic tubes in the magnetic field maintain their integrity as they extends along the field lines, i.e. there exist two families of surfaces, $\alpha(x$, $y, z)=c_{1}$ and $\beta(x, y, z)=c_{2}$, for which surface intersections give all field lines. However, the assumption is not true in 
general (see the following section). In a strictly mathematical sense, setting the relationship $\boldsymbol{B}=\nabla \alpha \times \nabla \beta$ or $\boldsymbol{A}=\alpha \nabla \beta$ is dubious, although it is true for the simpler magnetic fields.

It is worth noting that for a given magnetic field, people often use the relation $\boldsymbol{B} \cdot \Delta S=\Delta \alpha \Delta \beta$ to establish an $\alpha$ - $\beta$ grid on a surface $S$ that crosses the field lines, then map the values of $\alpha$ and $\beta$ on the surface along the field lines into the space. We note that in doing so the relation $A=\alpha \Delta \beta$ might not be explicitly considered.

Unlike Northrop and Teller's paper [3], in which Euler potentials were used for time-dependent magnetic field, the magnetic fields in most studies applying Euler potentials are time-independent. People start with setting $\boldsymbol{A}=\alpha \nabla \beta$, introducing $\boldsymbol{A} \cdot \boldsymbol{B}=0$ as a new gauge (e.g. [4,5]). However, no one imposes the restricted gauge transformation condition, such as those associated with the Lorentz gauge or Coulomb gauge.

We also note another related question that, if the vector potential $\boldsymbol{A}$ can be represented by Euler potentials $\alpha$ and $\beta$, then an electromagnetic field would be described with just three parameters $\alpha, \beta$ and $\phi$ instead of the four parameters $A_{x}, A_{y}, A_{z}$ and $\phi$. The physics of this seems unclear.

\section{An example where $\alpha$ and $\beta$ are inapplicable in the presence of field line-aligned current}

In the presence of field line-aligned currents, a magnetic field might be divided into several zones, and the field lines in different zones could spiral in different ways. Across the boundary surfaces of contiguous zones, the Euler potentials could be discontinuous, although the vector potential and magnetic field might not have discontinuities on those surfaces.

One example is shown in Figure 1. Three distinct families of field lines that define three disjoint zones result from the fields of two straight currents $I$ and $2 I$ embedded in a uniform background field $\boldsymbol{B}_{0}$ oriented in the same direction as the current. On the boundary surfaces between the contiguous zones, the field lines either side make contact but spiral upward in distinct ways. Hence, the field lines in each of the different zones must be defined by different Euler potential systems. Another similar result can be drawn for two currents flowing in opposing directions.

The discontinuity in Euler potential discussed above has major consequences on charged particle's drift motion. For the sake of simplicity, the magnetic field showed in Figure 1 is one non-confinement to the charged particle motion. In

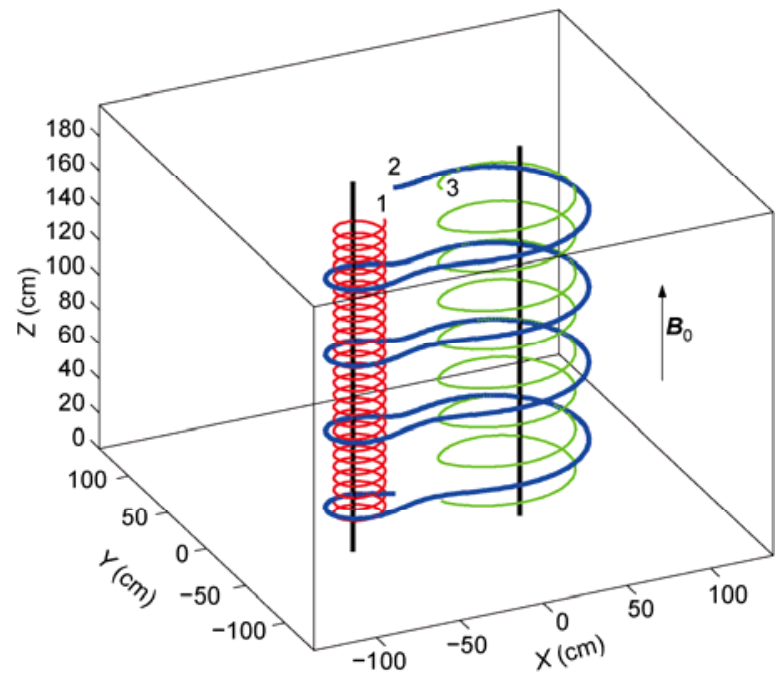

Figure 1 (Color online) Three families of field lines resulting from two straight current in a uniform $\boldsymbol{B}$ field.

the charged particle-confinement field, the Euler potential discontinuity such as one occurred in magnetic reconnection events makes particle drift complicated: the motion of particles limited inside each field line zone maintains relative independence, but particles drifting across the boundaries between the zones could lead to interactions of the plasmas located in these zones.

The present research at PMO was supported by the National Natural Science Foundation of China (10973043 and 41074107) and the National Basic Research Program of China (2011CB811402).

1 Grad H. Private communication to C. S. Gardner. Phys Rev, 1959, 115: 791-794

2 Grad H. Mathematical problems in magneto-fluid dynamics and plasma physics. In: Proceedings of the International Congress of Mathematicians, Stockholm, 1962. Djursholm: Institut Mittag-Leffler, 1963. 560-583

3 Northrop T G, Teller E. Stability of the adiabatic motion of charged particles in the Earth's field. Phys Rev, 1960, 117: 215-225

4 Ray E C. On the motion of charged particles in the geomagnetic field. Ann Phys, 1963, 24: 1-18

5 Stern D P. Euler potentials. Am J Phys, 1970, 4: 494-501

6 Le Sager P, Huang T S. Ionospheric currents and field-aligned currents generated by dynamo action in an asymmetric Earth magnetic field. J Geophys Res, 2002, 107: 1025-1038

7 D'Haeseleer W D, Hitchon W N G, Callen J D, et al. Flux Coordinates and Magnetic Field Structure. New York: Springer-Verlag, 1991. 241

8 Northrop T G. Adiabatic Motion of Charged Particles. New York: Intersciences Publishers Inc, 1963. 9

9 Phillips H B. Vector Analysis. New York: John Wiley \& Sons Inc, 1933. 104-106

Open Access This article is distributed under the terms of the Creative Commons Attribution License which permits any use, distribution, and reproduction in any medium, provided the original author(s) and source are credited. 